\title{
COMPENSATION EFFECT IN THE AMORPHOUS HYDROGENATED SILICON FROM POINT OF VIEW OF THE BARRIER-CLUSTER MODEL
}

\author{
Ivan BANÍK*, Jozefa LUKOVIČOVÁ*, Gabriela PAVLENDOVÁ*, Bimal Kumar SARKAR ${ }^{* *}$ \\ "Department of Physics, Faculty of Civil Engineering, Slovak University of Technology, \\ Radlinského 11, 81368 Bratislava, Slovak Republic, ivan.banik@stuba.sk \\ *** Department of Physics, School of Basic Applied \&Science, Galgotias University, Sector 17-A, \\ Greater Noida 201306, India
}

\begin{abstract}
Although hydrogenated amorphous silicon has its important practical application there still is not any generally accepted model explaining all the physical phenomena on-going in this matter. The aim of this paper is to familiarize the reader with a model allowing to explain the empirically observed compensation effect (Meyer-Neldel rule-MNR). This effect reflects the correlation between activation energy and pre-exponential factor in relation to the activation dependence of electrical conductivity of semiconductors, including a-Si:H. The model assumes that the recombination of carriers is conditioned by emission of series of monoenergetic phonons. The number of emitted phonons is dependent on the activation energy of disordered semiconductor, which influences the probability of recombination and thus the concentration of free electrons. Consideration of the relationship between compensation effect and Urbach rule is also presented.
\end{abstract}

Keywords: hydrogenated amorphous silicon, compensation effect, Meyer-Neldel rule, pre-exponential factor, hopping MNR

\section{INTRODUCTION}

Amorphous silicon, and its more useful alloy form, hydrogenated amorphous silicon (a-Si:H), has been the subject of investigation for over 30 years [1-3]. Research into a-Si began in England [4], but the major development, the demonstration of doping and the subsequent implementation of devices was made in Scotland by Spear and LeComber [4-7].

$\mathrm{A}-\mathrm{Si}: \mathrm{H}$ is a low-cost, efficient material which is used extensively for electronic devices. The advantages of a$\mathrm{Si}: \mathrm{H}$ are particularly evident when considering the photovoltaic application. The a-Si:H has a large optical absorption coefficient, the energy gap can be modulated to allow optimum conversion efficiency for sunlight. It can be alloyed with other elements (carbon, germanium) to create multijunction structures with increased energy conversion efficiency for sunlight.

The intrinsic disorder in amorphous silicon (a-Si) creates broken bonds, which negatively impact the electronic properties of materials. The main role of hydrogen in amorphous silicon is the passivation of the $\mathrm{Si}$ dangling bonds to restore a proper energy gap and the semiconducting properties, thus enabling extensive application of a-Si:H in the microelectronics and the photovoltaic industry. However, several questions are still unanswered.

The first report of hydrogenated amorphous silicon solar cells in 1976 [8] sparked worldwide interest as a promising economically viable source of renewable energy. The materials used in the a-Si:H based solar cells are in fact silicon hydrogen alloys which typically contain about 5 to $20 \%$ of hydrogen.

Serious problem of solar cells based on a-Si:H is so called Staebler-Wronski effect [9] associated with light induced degradation of the material. Continuous progress has been made over the years not only in improving the initial properties of a-Si:H based materials but also in reducing their light induced degradation.
Hydrogenated amorphous silicon is still the subject of intense research. Dark conductivity in a-Si:H can be described by two main processes. The first is the standard extended states conduction process. The second conduction process is referred to as variable-range hopping (VRH) conduction. Great attention is paid to the system a-Si / C: H, respectively a-C:H.

\section{MEYER-NELDEL RULE AND a-Si:H}

Semiconductors are usually characterized by the temperature dependence of their electrical conductivity, $\sigma$

$\sigma=\sigma_{0} \exp \left(-\frac{W}{k T}\right)$

where $\sigma_{0}$ is a constant and $W$ - activation energy [10]. Activation dependence (1) corresponds to the first of these mechanisms. For many classes of materials, especially amorphous hydrogenated silicon, chalcogenide glasses, and organic semi-insulators, experimental evidence suggests that a correlation exists between the activation energies and pre-exponential factors in the following form [11-15]

$$
\sigma_{0}=\sigma_{00} \exp (b W)
$$

or

$$
\ln \sigma_{0}=\ln \sigma_{00}+b W \text {, }
$$

where $b$ and $\sigma_{00}$ are constant. This relation can be written as

$\sigma_{0}=\sigma_{00} \exp \left(\frac{W}{k T_{M N}}\right), \quad \sigma_{0}=\sigma_{00} \exp \left(\frac{W}{E_{M N}}\right)$.

Here $b=1 / k T_{\mathrm{MN}}=1 / E_{\mathrm{MN}}$. The value $E_{\mathrm{MN}}=k T_{\mathrm{MN}}$ is the $\mathrm{MN}$-energy and $T_{\mathrm{MN}}$ the MN-temperature. Equation (2) is often referred to as the compensation rule, or Meyer- 
Neldel empirical rule (MNR). Constant $\sigma_{00}$ is often called the Meyer-Nedel pre-exponential factor and $E_{\mathrm{MN}}=k T_{\mathrm{MN}}$ the Meyer-Neldel characteristic energy.

For electric conductivity of above mentioned group of substances holds $(1,3)$

$$
\sigma=\sigma_{00} \exp \left(\frac{W}{k T_{M N}}\right) \exp \left(-\frac{W}{k T}\right) \text {. }
$$

An a-Si:H doped with different impurities, which affect the activation energy, shows such dependence (Fig. 1). Amorphous hydrogenated carbon a-C:H and systems of a-Si / C:H have similar behaviour.

MN rule holds in disordered materials when $W$ varies by doping, by surface absorption, light soaking or by preparing films under different conditions. This rule has also been observed for liquid semiconductors and fullerens. The validity of the $\mathrm{MN}$ rule has also been reported in the case of chalcogenide glasses. In the case of these glasses this rule is observed by variation of $W$ on changing the composition of the glassy alloys in a specific glassy system.

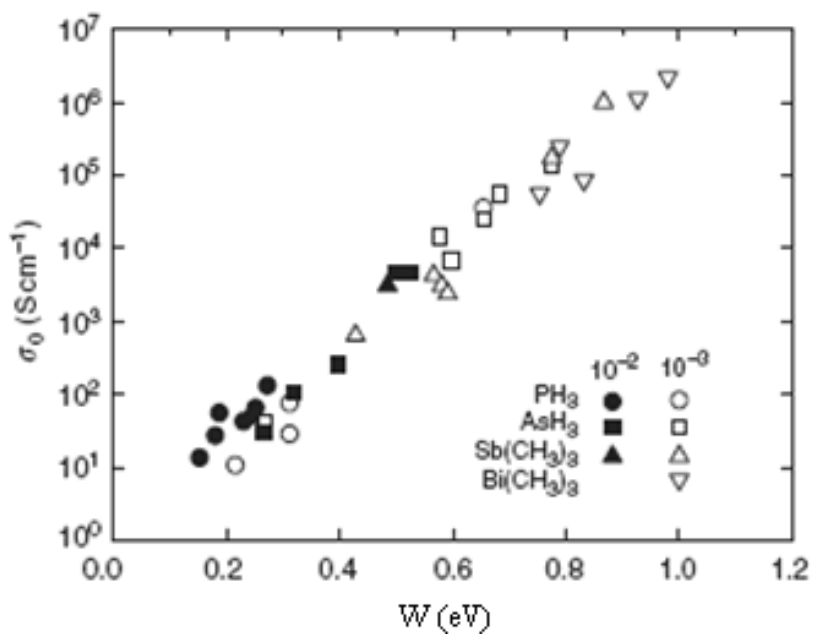

Fig. 1 The pre-exponential factor $\sigma_{0}$ plotted as a function of activation energy $W$ for a-Si:H doped with various dopants. The fitting of the experimental data (rel.3) produces $\mathrm{E}_{\mathrm{MN}}=67 \mathrm{meV}$ and $\sigma_{00} \approx 1 \mathrm{Scm}-1$ [16]

\section{AN EXPLANATION OF THE MNR}

In the following we will present some notes on one possible explanation of MNR, which was published in the works $[11,12]$. In our barrier-cluster model it is assumed, that activation energy of a semiconductor influences the recombination process of current carriers. An increase of activation energy $W$ of a non-crystalline semiconductor decreases the probability of carrier recombination. This process necessary influences equilibrium concentration of conduction electrons (carriers) and subsequently electric conductivity of the semiconductor. As a result we obtain the relation identical with that one representing empirical compensation effect - Meyer-Neldel rule.

\subsection{Dependence of conductivity on activation energy}

A transition of an electron from conduction band to valence band in a non-crystalline semiconductor proceeds in our case predominantly by production of phonons. The total energy of produced phonons will correspond to that one released in electron transition. Further we shall assume that in a substance under consideration dominates phonon production, which average energy is $\Delta E$. It means that phonon production of other phonons is negligible. At the transition of an electron from conduction band to valence band gained energy $2 W$ is used in production of $N$ phonons each of them has energy $\Delta E$ so that $2 W=N \Delta E$ or

$N=2 W / \Delta E$.

Let $w_{1}$ be the probability of production of one phonon which energy is equal to $\Delta E$. Probability $w_{N}$ of production of $N$ phonons of equal energy $\Delta E$ due to the electron lattice interaction will be

$w_{N}=\left(w_{1}\right)^{\mathrm{N}}$

If we write down probability $w_{1}$ as

$w_{1}=\exp \left(-\varepsilon_{1}\right)$

where $\varepsilon_{1}$ is a positive value, then probability $w_{\mathrm{N}}$ one can write with respect to $(6,7)$ as

$w_{\mathrm{N}}=\exp \left(-N \varepsilon_{1}\right)=\exp \left(-\varepsilon_{1} 2 W / \Delta \mathrm{E}\right)=\exp (-b W)$,

where the constant $b$ is given by

$b=2 \varepsilon_{1} / \Delta E$.

Relation (8) gives at the same time the probability of recombination; it means the transition probability of an electron from conduction to valence band. That one is proportional to the probability of production of $N$ phonons and also proportional to the $\exp (-b W)$. With an increase of activation energy probability of recombination according to (8) exponentially decreases.

\subsection{Equilibrium concentration of conduction electrons}

A number of free electrons generated in a unit time is given as

$G=\left(\frac{d n}{d t}\right)_{\text {recom }}=C_{1} \exp \left(-\frac{W}{k T}\right)$,

where $C_{1}$ is a constant. For a recombination process we suppose (in accordance with the relation (8)) that the relation

$R=\left(\frac{d n}{d t}\right)_{\text {recom }}=n C_{2} \exp (-b W)$

is valid. Here $C_{2}$ is a constant. The number of recombinations in a unit of time is proportional to the number $n$ as well as to the probability (8) of production of $N$ phonons at the electron transition. In equilibrium state is valid $R=G$, so that

$C_{1} \exp (-W / k T)=n \cdot C_{2} \exp (-b W)$ 
From that relation follows for equilibrium concentration $n$ of free carriers

$n=\mathrm{C}_{00} \exp (b W) \exp (-W / k T)$

where $C_{00}$ is determined by the constants $C_{1}$ and $C_{2}$.

\subsection{Electric conductivity}

It is known that electric conductivity $\sigma$ is proportional to $n: \sigma \approx n$. If mobility of carriers is independent on activation energy $W$ (or that dependence is negligible) and if we put $b=1 / k T_{\mathrm{MN}}$, one can write with respect to (13)

$\sigma=\sigma_{00} \exp \left(\frac{W}{k T_{M N}}\right) \exp \left(-\frac{W}{k T}\right)$

and for the pre-exponential factor

$\sigma_{0}=\sigma_{00} \exp \left(\frac{W}{k T_{M N}}\right)$,

which expresses the conventional compensation effect or Meyer-Neldel rule (3).

\section{HOPPING CONDUCTION AND "HOPPING MNR"}

At low enough temperatures, variable-range hopping (VRH) in the localized states is expected to become predominant as the number of free carriers in the band decreases. Assuming a constant density of states $N\left(E_{\mathrm{F}}\right)$ within $k T$ of the Fermi level, Mott [7] obtained for VRH,

$\sigma=\sigma_{0} \exp \left(-\left(\frac{T_{H}}{T}\right)^{1 / 4}\right)$.

Here, $\sigma_{0}$ and $T_{\mathrm{H}}$ are functions of $N\left(E_{\mathrm{F}}\right)$. Eq. (16) is widely known as Mott's $T^{-1 / 4}$ law of VRH.

It was empirically found [17] that many semiconductors including a-Si:H, in the area of hopping conduction follow a new MNR - hopping MNR, namely

$\sigma_{0}=\sigma_{00} \exp \left(\left(\frac{T_{H}}{T_{H M N}}\right)^{1 / 4}\right)$,

where, in analogy with $1 / E_{\mathrm{MN}}$ of the conventional MNR, we have defined $\left(1 / T_{\mathrm{HMN}}\right)^{1 / 4}$ as the slope of the straight line (3), in the hopping MNR plot.

In the previous we have presented a possible explanation for the conventional MNR (2, 3). Concerning hopping MNR, there is no available explanation. In this paper we would like to show some formal parallel concerning two empirical relationships. These relationships are indeed quite different, corresponding to the two different mechanisms of electrical conductivity, but anyhow there is some similarity. Let us look at the equations

$\sigma=\sigma_{00} \exp \left(\frac{W}{E_{M N}}\right) \exp \left(-\frac{W}{k T}\right)$, $\sigma_{0}=\sigma_{00} \exp \left(\left(\frac{T_{H}}{T_{\text {HMN }}}\right)^{1 / 4}\right) \exp \left(-\left(\frac{T_{H}}{T}\right)^{1 / 4}\right)$.

Comparing both relations $(18,19)$ we see that the role of activation energy $W$ in the second relation was taken by variable $T_{\mathrm{H}}$ and $E_{\mathrm{MN}}$ was replaced by variable $T_{\mathrm{HMN}}$. Exponential expressions on the right sides of both equations represent the character of temperature dependence of electrical conductivity at different conduction mechanisms. Exponential expressions in terms of pre-exponential factors (first from left) are in analogical way "linked" to an exponential expressions figuring after them.

The analogy of two relations offers the idea that both types of MNR (conventional MNR and hopping MNR) are aroused by some analogous mechanism. By the hopping conductivity - as it is commonly presented in literature - it is about processes taking place close to the Fermi level $E_{\mathrm{F}}$. In this case, activation energy is not involved into the process. On the other hand, it is generally accepted that electron hopping cannot take place without the participation of phonons and the phonons mean energy. Therefore, some authors are talking about the "hopping energy", respectively the level at which hopping takes place. But so far there is no uniform view on this issue.

Theoretically, the value $T_{\mathrm{H}}$ in relation (19) could be related with the ability of the grid to absorb e.g. impulse of an electron after hopping-skipping to a new position at the free level in the mobility gap. We have in mind the probability of such absorption. The question is: will this "impulse absorption" go on in certain doses as it is by the absorption of energy $2 W$ by conventional MNR? Is it not impulse, respectively quaziimpulse hidden by variable $T_{\mathrm{H}}$ ? Variable $T_{\mathrm{HMN}}$ might be then connected with the grid ability to absorb change of the impulse and thus actually sending compensation phonons. These are however only our questions.

Notes: Physical properties of disordered semiconductors are the topic of the works [18-25]. The explanation of some electrical and optical properties of disordered semiconductors based on barrier-cluster model was published in [11,12,26-30]. In the paper [26] was published "Band model of diffusion", that allows to explain compensation effect in the diffusion and diffusionrelated processes, which include catalysis, crystallization.

\section{MNR AND OPTICAL ABSORPTION}

In this paragraph we will be interested in the optical absorption in the area of exponential tail, where Urbach's rule holds.

\subsection{The Urbach's rule}

The Urbach's rule is an empirical rule, describing the absorption of light in exponential tails of optical absorption spectra [5-7,23]. It expresses the dependence of the coefficient of the optical absorption $\alpha$ on the photon energy $h f$ and the temperature $T$. It can be expressed mathematically by the relation 


$$
\alpha=C \exp \left(\gamma \frac{h f-E}{k T}\right)
$$

or equivalently as

$$
\ln \alpha=\left(\gamma \frac{h f-E}{k T}\right)+\text { const . }
$$

Thus, $\ln \alpha(h f)$ is a straight line, here $\gamma, E$ are parameters and $k$ is the Boltzmann constant. This relation holds for relatively high temperatures. At low temperatures, the absorption is given by a relation similar to $(20,21)$ but the real temperature $T$ has to be replaced by a constant parameter $T^{*}$. This point was not explained satisfactorily up to now $[5,18,23]$.

\section{AN ATEMPT TO EXPLAIN THE URBACH'S RULE}

The Urbach's rule can be explained on the base of the barrier-cluster model assuming that an electron during the optical transition from the conduction into valence band can absorb both the energy of a photon and of a phonon [27-30]. Then the energy increase of the electron is equal to $h f+W_{\text {phon }}$, where $W_{\text {phon }}$ is the energy gained by the electron from lattice vibrations. The photon energy is given by the frequency of light. The phonon energy has a statistical character. If the photon energy $h f<2 W$ is less than the width of the forbidden gap then the absorption of light cannot happen. But, the absorption can come into being if the missing energy $2 W-h f$ needed for the transition is supplied by a phonon. This is the energy, which an electron needs in order to equalize its energy with the width of the forbidden gap.

The probability of occurrence of phonons with an energy higher than or equal to $2 W-h f$ is proportional, at temperature $T$, to the expression

$$
p=C \exp \left(-\frac{2 W-h f}{2 k T}\right)
$$

The probability of optical absorption (with the participation of phonons) is proportional to the same expression. The absorption coefficient $\alpha$ in an energy region of photons less than the width of the forbidden gap can be determined as

$$
\alpha=C \exp \left(\frac{h f-2 W}{2 k T}\right)=C \exp \left(\gamma \frac{h f-2 W}{k T}\right)
$$

where $\gamma=1 / 2, C$ is a constant. Thus

$$
\ln \alpha=\left(\frac{h f-2 W}{2 k T}\right)+\text { const . }
$$

This relation represents a straight line (exponential tail of optical absorption), with a slope decreasing with increasing the temperature $T$. This behaviour corresponds to the one known with disordered semiconductors (if $E=$ $2 W)$. However, it has become known from experiments that the situation changes with the decrease of the temperature. The slope of the straight lines given by (24) continues being unchanged with temperature at low temperatures. Only a parallel shift to the lower absorption of "exponential tails" is observed.

The relation (23) is no longer valid. At lowering the temperature of a non-crystalline semiconductor, the parameter $T$ in relation (23) no longer represents temperature. It seems as if the temperature was freezed at a certain value of $T^{*}$. The essence of this phenomenon is not reliably explained till now [1], but we will try to clarify it partially. Let us note that the parameter $\gamma$ is (experiments) about $0.4-0.55$ [6,7] in the chalcogenide glasses. When comparing (20) and (23), we see that the (barrier-cluster model) yields the constant $\gamma=0,5$. This may be considered as a good agreement in the research of non-crystalline semiconductors.

Remarks: We cannot agree with the idea that the exponential tails are caused by the distribution of density of states in the mobility gap. Of course, the possible density of states can to some extent influence exponential tails, but we do not consider it the primary cause of such tails. We consider the participation of phonons in absorption the primary cause of tail formation. By this it is easy to explain "universality" of tails - exponential character and also practically the same slope in a rich variety of semiconducting materials. Something like this cannot be provided by the density of states of various and varied materials. In our interpretation, the distribution density of states is a secondary factor. This is in agreement with the characteristics of a-Si, respectively a$\mathrm{Si}: \mathrm{H}$, in which the standard exponential tails appear just after the elimination of free bonds, thus after the hydrogenation. Thus the tails appear after the forbidden zone purification.

\subsection{MNR and Urbach's rule - consideration and questions}

The basic idea is subsequent. As it has been said above, the energy released at non-radiative recombination of $e-h$ pairs is transformed into the energy of monoenergetic phonons. These phonons create a phonon field in a semiconductor. At higher temperatures this additional phonon field is relatively "weak" and that is why its effect does not appear significant - thermal phonons dominate. At higher temperatures, the relation (23) holds, where $T$ denotes the temperature. The slope of a straight line $\ln \alpha(h f)$ decreases with the increase of the temperature.

At lower values of $k T$, it will be necessary to accept the substitution

$k T+k T^{*}=E_{\mathrm{U}}$,

instead of $k T$. The second term, $k T^{*}$, represents effective energy of mono-energetic phonons. It will be valid that

$\alpha=C \exp \left(\gamma \frac{h f-2 W}{E_{U}}\right)=C \exp \left(\gamma \frac{h f-2 W}{k T+k T *}\right)$.

By lowering the temperature, the first term $k T$ becomes small and the second term $k T^{*}=E_{U}^{*}$ begins to dominate. By a subsequent cooling, the relation achieves the limiting form 


$$
\alpha=C \exp \left(\gamma \frac{h f-2 W}{E_{U}^{*}}\right)
$$

A result is that the slope of the exponential tails (in the straight line representation) by lowering temperatures will gradually become smaller. It looks as if at very low temperatures the tail of optical absorption is shifted almost parallel to the lower absorption. The limiting straight line will be the one, which corresponds to the exponential function (26). The question remains: Can the considered effect be the major cause of Urbach's rule in the region of low temperatures?

Remarks: The described effect of monoenergetic phonons (produced in non radiative recombination), the optical absorption is in principle certainly possible, and real. An open question is the extent of such an effect. It is possible that under normal conditions, the effect is negligible and that it occurs noticeably only at sufficiently low temperatures and high light intensity. We also cannot exclude certain "resonance effect" by absorption of monoenergetic phonons that was produced before by considered system alone. Questions remain.

\section{CONCLUSIONS}

Electrical conductivity in a-Si:H can be described by two main processes. The first is the standard extended states conduction process. The second conduction process is referred to as variable-range hopping (VRH) conduction. Validity of the conventional Meyer-Neldel rule in non-crystalline semiconductors is in presented paper explained assuming that recombination transition of an electron from conduction band to valence band is conditioned by emission of a number of monoenergetical phonons. Probability of such complex process is determined by number of emitted phonons. This probability exponentially decreases with increasing number of phonons and consequently with the width of the forbidden band. The probability of recombination influences equilibrium concentration of free electrons in conduction band and subsequently electric conductivity. In such a way we have finally obtained the relation which is equivalent to compensation effect, or the Meyer-Neldel rule. By lower temperatures in disordered semiconductors the hopping transport dominates. In this type of electrical conductivity specific "hopping MNR" is experimentally observed.

It is shown in the paper that monoenergetic phonons can influence optical absorption. This enables explaining of Urbach's rule in low temperature area.

\section{ACKNOWLEDGEMENT}

At the end, we would like to express our thanks to Prof. Viktor Bezák from Comenius University in Bratislava and Prof. František Čulík from the Faculty of Civil Engineering, Slovak University of Technology in Bratislava for valuable discussions on this topic.

\section{REFERENCES}

[1] LEWIS, A. J. - CONNELL, G. A. N. - PAUL, W. PAWLIK, J. R. - TEMKIN, R.: Hydrogen incorporation in amorphous germanium, AIP Conf. Proc. 20 p.27-32, (1974).

[2] LECOMBER, P. G. - LOVELAND, R. J. - SPEAR, W. E. - VAUGHAN, R. A.: in: STUKE, J. BRENIG, W. (Eds.), Amorphous and Liquid Semiconductors, Taylor and Francis, London, 1974, p. 245, (1974).

[3] BEYER, W. - STUKE, J.: Amorphous and Liquid Semiconductors, in: STUKE, J., BRENIG, W. (Eds.), Taylor and Francis, London, 1974, p. 251, (1974).

[4] SPEAR, W. - LECOMBER, P.: Substitutional doping of amorphous silicon, Solid State Commun. 17, p.1193, (1975).

[5] GASPARI, F.: Kap. 1, Optoelectronic Properties of Amorphous Silicon the Role of Hydrogen: From Experiment to Modeling , Optoelectronics Materials and Techniques, Ed. by Padmanabhan Predeep, ISBN 978-953-307-276-0, p. 484, Publisher: InTech, (2011).

[6] BRODSKY, M. H.: Amorphous semiconductors, Springer Verlag Berlin, Heidelberg, New York 1979.

[7] MOTT, N.: Conductivity, Localization, and the Mobility Edge, in The Physics of Hydrogenated Amorphous Silicon, Vol. II, Eds. J.D. Joannopoulos \& G. Lucovski, Springer-Verlag, ISBN 0387128077, New York (1983).

[8] CARLSON, D. E. - WRONSKI, C. R.: Amorphous silicon solar cell, Appl. Phys. Lett. Vol. 28, p. 671673, (1976).

[9] STAEBLER, D. L. - WRONSKI, C. R.: Reversible conductivity changes in discharge-produced amorphous Si, Appl. Phys. Lett. 31, p. 292-294, (1977).

[10] MEYER, W. - NELDEL, H.: Electrical conductivity in the polycrystalline $\mathrm{ZnO}, \mathrm{Z}$. Tech. Phys. (Leipzig), 18, p. 588, (1937).

[11] BANÍK, I.: Recombination processes, Meyer-Neldel rule and non-exponential decay in disordered semiconductors, Journ. of Optoelectron. and Adv. Materials, Vol. 13, No. 11-12, p. 1364-1376, (2011).

[12] BANÍK, I.: One way to explain the Meyer-Neldel rule, Chalcogenide Letters, Vol. 6, No.12, p. 629633, (2009).

[13] BOISVERT, G. - LEWIS, L. J. - YELON, A.: Many-body nature of the Meyer-Neldel compensation law for diffusion, Phys. Rev. Lett. 75, p. 469, (1995).

[14] YELON, A. - MOVAGHAR, B. - BRANZ, H. M.: Origin and consequences of the compensation 
M(eyer-Neldel) law, Phys. Rev. B 46, 12244 -12250, (1992).

[15] BRANZ, H. M. - YELON, A. - MOVAGHAR, B.: Physics of the Meyer-Neldel Rule in Amorphous Silicon, Mat. Res. Soc. Symp. Proc. 336, p. 159, (1994).

[16] CARLSON, D. E. - WRONSKI C. R.: Amorphous semiconductors, Topis in Appl. Phys. Vol. 36, Ed. BRODSKY, M. H. (New Yourk: Springer), p. 287329, (1979).

[17] DALVI, A. - REDDY, N. P. - AGARWAL, S. C.: The Meyer-Neldel rule and hopping conduction, Solid State Communications 152, p. 612-615, (2012).

[18] STREET, R. A.: Hydrogenated Amorphous Silicon, Cambridge Univresity Press, Cambridge, p. 413, (1991)

[19] ABTEW, T. A. - ZHANG, M. L. - YUE PAN DRABOLD, D. A.: Electrical conductivity and Meyer-Neldel rule: The role of localized states in hydrogenated amorphous silicon, Journal of NonCrystalline Solids 354, p. 2909-2913, (2008).

[20] SHIMAKAWA, K. - ABDEL-WAHAB, F.: The Meyer-Neldel rule in chalcogenide glasses, Appl. Phys. Lett. 70, p. 652, (1997).

[21] ELLIOT, S. R.: Theory of ac conduction in chalcogenide glasses, Philos. Mag. B 36, p. 12911304, (1977).

[22] GASPARI, F. - SHKREBTII, A. - KUPCHAK, I. PERZ, J. M.: Modeling noncrystalline materials: Use of vibrational spectra as a protocol for validation, Phys. Rev. B Vol 79, p. 224203, (2009).

[23] POPESCU, M.: Physics and Applications of Disorde-red Materials, INOE Publishing House, (2002).

[24] OVERHOF, H. - THOMAS, P.: Electronic Transport in Hydrogenated Amorphous Semiconductors, Springer-Verlag, Berlin, (1989).

[25] POPESCU, M.: The closed cluster model and the charged coordination defects in chalcogenide glasses, J. Optoelectron. Adv. Mater. 6(4), p. 1147, (2004).

[26] BANÍK, I.: Band model of diffusion and the MeyerNeldel rule, Journal of Non-Oxide Glasses Vol. 1, No. 4, p. 267-275, (2009).

[27] BANÍK, I.: Optical properties of the hydrogenated amorphous silicon from point of view of the barriercluster model, Journ. of Non-Oxide Glasses, Vol.2, No. 2, p. 107-120, (2010).

[28] BANIK, I.: Photoconductivity in chalcogenide glasses on the base of the barrier-cluster model, Journ. of Optoelectr. and Adv. Mater., Vol.11, No. 12, p. 1931-1945, (2009).
[29] BANIK, I.: Barrier-cluster model - the base for understanding of the optical phenomena in noncrystalline semiconductors, Journ. of Optoelectr. and Adv. Mater., Vol. 11, No. 12, p. 1915-1930, (2009).

[30] BANÍK, I.: Photoconductivity in chalcogenide glasses in Non-Stationary Regime, Acta Electrotechnica et Informatika, Vol. 10, No. 3, p. 52-58, (2010).

Received February 9, 2013 , accepted February 15, 2013

\section{BIOGRAPHIES}

Ivan Baník Graduated in 1958, CSc. in 1982, assoc. professor in 1986, full professor in 2006. His main interests: Non-crystalline semiconductors, Optical properties of disordered materials, Thermophysics, High pressure physics, Didactics. Places of employment: Gymnasium in B. Bystrica (1958-61), Training College in B. Bystrica (1961-64), Electrotechnical faculty SUT in Bratislava (1964-76), Faculty of Civil Engineering SUT in Bratislava (1976-2012).

Jozefa Lukovičová Graduated in 1970 - Comenius University in Bratislava, CSc. (PhD) in 1985, Assoc. prof. in 1997. Main interests: Thermophysics, Physical properties of the building porous materials, Noncrystalline semiconductors, Didactics. Places of employment: Slovak Academy of Sciences in Bratislava (1970-1978), Department of Physics, Faculty of Civil Engineering, SUT Bratislava (1979-2012).

Gabriela Pavlendová Graduated in 1980 - Faculty of Electrotechnical Engineering SUT, Complementary Pedagogical studies 1992, Pedagogical University in Nitra, Faculty of Natural Sciences -1995, PhD (CSc.) in 2003. Main interests: Non-crystalline semiconductors, Thermophysics, Didactics. Places of employment: Slovak Academy of Sciences in Bratislava (1980-83), Trnava Motor Factory (1983-87), Slovak University of Technology, Mechanical Engineering Faculty (1998-99), Secondary Grammar School in Bratislava (1991-2009), University of Comenius in Bratislava, External Lecturer (1999-present), Faculty of Civil Engineering, SUT in Bratislava (2009-2012).

Bimal Kumar Sarkar Professor, Department of Physics, School of Basic \& Applied Sciences, Galgotias University Sec. 17-A, Greater Noida 201306, India. Interested in thermal and optical properties investigations of solids, such as the structure, transport, magnetism, thermal, optical properties, and studies in some diluted magnetic semiconductors, specially ternary or quaternary alloys. Interested in various NMR methodologies to study the broad range of problems in chemistry, bio-physics, and materials science. 